\title{
A role of diffusion tensor imaging in movement disorder surgery
}

\author{
Garni Barkhoudarian • Tony Klochkov • Mark Sedrak • \\ Andrew Frew • Alessandra Gorgulho • Eric Behnke • \\ Antonio De Salles
}

Received: 8 April 2010 / Accepted: 8 July 2010 /Published online: 21 July 2010

(C) The Author(s) 2010. This article is published with open access at Springerlink.com

\begin{abstract}
The safe and reversible nature of deep brain stimulation (DBS) has allowed movement disorder neurosurgery to become commonplace throughout the world. Fundamental understanding of individual patient's anatomy is critical for optimizing the effects and side effects of DBS surgery. Three patients undergoing stereotactic surgery for movement disorders, at the institution's intraoperative magnetic resonance imaging operating suite, were studied with fiber tractography. Stereotactic targets and fiber tractography were determined on preoperative magnetic resonance imagings using the Schaltenbrand-Wahren atlas for definition in the BrainLab iPlan software (BrainLAB Inc., Feldkirchen, Germany). Subthalamic nucleus, globus pallidus interna, and ventral intermediate nucleus targets were studied. Diffusion tensor imaging parameters used ranged from 2 to $8 \mathrm{~mm}$ for volume of interest in the $x / y / z$ planes, fiber length was kept constant at $30 \mathrm{~mm}$, and fractional anisotropy threshold varied from 0.20 to 0.45 . Diffusion tensor imaging tractography allowed reliable and reproducible visualization and correlation between frontal eye field, premotor, primary motor, and primary sensory
\end{abstract}

Presented at the American Society of Stereotactic and Functional Neurosurgery meeting, Vancouver, 2008

G. Barkhoudarian $(\bowtie) \cdot$ T. Klochkov $\cdot$ M. Sedrak • A. Gorgulho • E. Behnke $\cdot$ A. De Salles

Department of Neurosurgery, David Geffen School of Medicine, University of California Los Angeles (UCLA),

Room 18-228 NPI, BOX 957039, Los Angeles, CA 90095-7039, USA

e-mail: gbarkhoudarian@mednet.ucla.edu

\section{A. Frew}

Department of Neurology, David Geffen School of Medicine, University of California Los Angeles (UCLA),

Room 18-228 NPI, BOX 957039, Los Angeles, CA 90095-7039, USA cortices via corticospinal tracts and corticopontocerebellar tracts. There is an apparent increase in the number of cortical regions targeted by the fiber tracts as the region of interest is enlarged. This represents a possible mechanism of the increased effects and side effects observed with higher stimulation voltages. Currently available diffusion tensor imaging techniques allow potential methods to characterize the effects and side effects of DBS. This technology has the potential of being a powerful tool to optimize DBS neurosurgery.

Keywords Deep brain stimulation .

Diffusion tensor imaging - Fiber tractography . $\mathrm{STN} \cdot \mathrm{GPi} \cdot \mathrm{ViM}$

\section{Introduction}

Deep brain stimulation (DBS) has tremendously changed the field of functional neurosurgery from one of ablative techniques to adjustable and reversible applications. While DBS has revolutionized the treatment of various brain disorders, the mechanisms of side effects, such as eye deviation, dysarthria, face, and hand activation are coming to light.[15]

The application of diffusion tensor magnetic resonance imaging has enhanced the ability to view anatomical detail beyond what is seen by conventional magnetic resonance imaging (MRI) or computed tomography scans. Indeed, diffusion tensor imaging (DTI) allows in vivo imaging of fiber tracts in humans.[1, 12, 14] Water diffusion in white matter is directionally dependant, allowing the formation of anisotropic maps and evaluation of their movement vectors. Fiber tracts (e.g., axons) can be deduced by calculating the cumulative molecular water diffusion vectors. When there 
is no net diffusion, as in some gray matter regions or crossing white matter tracts, the region may be isotropic and fiber tracking in these regions with this technology is not possible. This type of imaging has been validated with known fiber tracts and animal models.[6, 13, 16]

In this study, we sought to better understand the mechanisms of side effects from DBS in patients undergoing treatment for movement disorders.

\section{Methods}

After institutional review board approval, we retrospectively reviewed 18 sequential MRI image sets of patients who underwent DBS for movement disorders between March and July 2007. Most of these patients had DTI imaging preoperatively performed in the 1.5 Tesla Sonata intraoperative MRI (iMRI) suite (Siemens AG, Munich, Germany) at the UCLA Medical Center. Medtronic 3389 Deep Brain Stimulator Leads (Medtronic, Minneapolis, $\mathrm{MN})$ were placed in all patients. Diffusion tensor source imaging acquisition was undertaken before placement of the Leksell stereotactic frame (Elekta Instrument AB, Stockholm, Sweden) that was placed parallel to Reid's line under propofol sedation. A detailed description of the stereotactic procedure, as well as the imaging acquisition for targeting, had been previously reported.[3,9]

The side effects obtained during surgery were registered to all patients and their respective targets. During surgery, the threshold for clinical improvement was noted and the amplitude of electrical stimulation was increased until the side effects appeared. Thresholds for side effects were noted for each patient. The protocol for intraoperative stimulation in our institution was previously reported.[2, 3, $5,9,11,15]$

To be included in this study, we evaluated the preoperative MRI sequences to be certain that the entire brain was scanned with high-quality DTI fractional anisotropy maps for determination of white-matter fiber tracts. Patients who had the devices removed postoperatively due to infection or malfunction were excluded. Patients who were lost to follow-up or did not have postoperative recording data were excluded. After screening these 18 patients for the inclusion criteria, 5 were deemed appropriate for analysis and 3 were chosen to for a detailed performance of fiber tracking on the three different targets commonly selected for deep brain stimulation (DBS) cases, namely, subthalamic nucleus (STN), globus pallidus interna (GPi), and ventral intermediate nucleus (VIM).

The three patients selected for this report had 12directional DTIs performed before placement of the frame. DTI data were acquired using single-shot spinecho echo-planar imaging with $\mathrm{TR}=10,000 \mathrm{~ms}$, $\mathrm{TE}=$
$90 \mathrm{~ms}$, acquisition matrix $=128 \times 128$ and field of view $=$ $25.6 \mathrm{~cm}$. Using a slice thickness of $2 \mathrm{~mm}$ with no gap, diffusion-sensitizing gradient encoding was applied in 12 directions by using a diffusion-weighted factor $b=700$ $\mathrm{s} / \mathrm{mm}^{2}$, and one image was acquired without use of a diffusion gradient, i.e., $b=0 \mathrm{~s} / \mathrm{mm}^{2}$. The DTI imaging time was approximately $4 \mathrm{~min}$.

BrainLab's iPlan Stereotaxy 2.6 software (BrainLAB Inc., Feldkirchen, Germany) was used in all the analyses. The preoperative DTI sequences were fused with the postoperative T1 sequences. Final electrode placement was analyzed postoperatively, and final trajectories were traced based on artifact location using postoperative magnetic resonance scans.[3] The measuring tool was then used to pinpoint the location of the desired lead by using probe view and centering the location at the appropriate distance. Due to data acquisition limitations, the smallest region of interest (ROI) attainable was a $2-\mathrm{mm}$ cube.

Patient 1 underwent DBS of the STN for Parkinson disease treatment. Fiber tractography was performed at right lead 1 (Fig. 1a) where improvement in motor function was seen at $1.5 \mathrm{~V}$. The threshold for uncontrollable arm

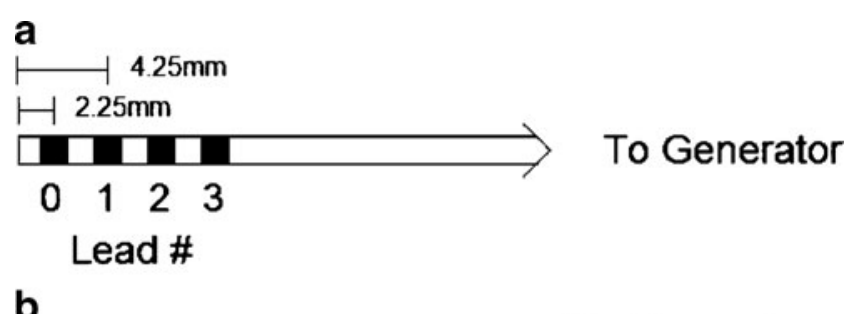

To Generator

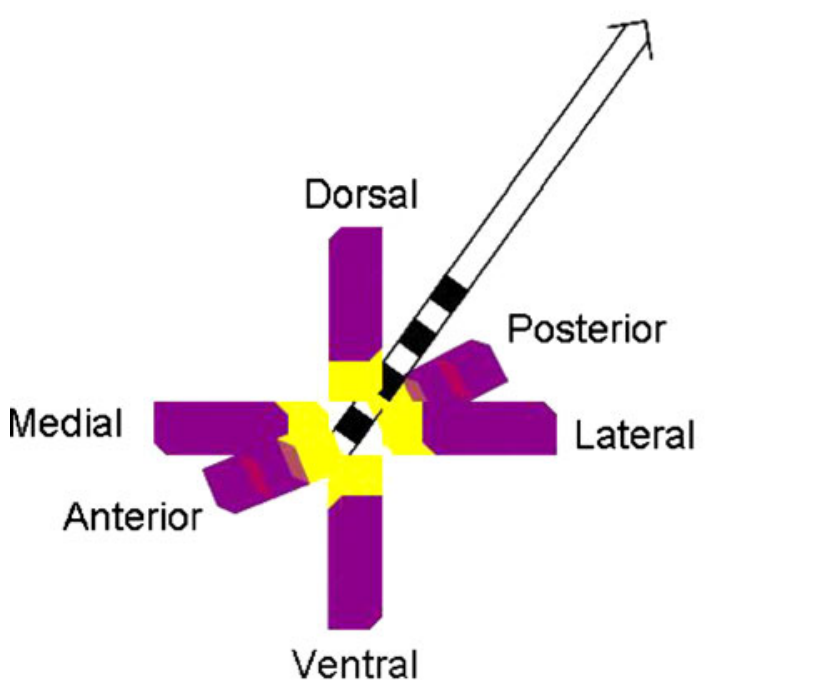

Fig. 1 a Diagram of the Medtronic 3389 intracranial DBS lead orientation and measurements. b Color-coded diagram of ROI voxels used for fiber tractography at specific locations surrounding the case electrode lead. The yellow boxes demonstrate the $2 \times 2 \times 2 \mathrm{~mm}$ small ROIs. The purple boxes demonstrate the total of $2 \times 2 \times 8 \mathrm{~mm}$ large ROI (including the original $2 \times 2 \times 2 \mathrm{~mm}$ ROI). Fiber tractography demonstrated in Figs. 3-5 adhere to this color scheme 
movement was noted at $3.4 \mathrm{~V}$. Final coordinates for the tip of the electrode were $10.6 \mathrm{~mm}$ lateral to the $\mathrm{AC} / \mathrm{PC}$ line, $1.6 \mathrm{~mm}$ posterior to the $\mathrm{AC} / \mathrm{PC}$ midcommisural plane, and $3.6 \mathrm{~mm}$ ventral to $\mathrm{AC} / \mathrm{PC}$ plane.

Patient 2 underwent DBS of the ventral intermediate nucleus (VIM) for treatment of essential tremor. Fiber tractography was performed at left lead 0 (Fig. 1a), where tremor arrest was noted at $1.8 \mathrm{~V}$. Facial pulling and dysarthria was noted at $3.6 \mathrm{~V}$. Final coordinates for the tip of the electrode were $11.3 \mathrm{~mm}$ lateral to the $\mathrm{AC} / \mathrm{PC}$ line, $8.14 \mathrm{~mm}$ posterior to $\mathrm{AC} / \mathrm{PC}$ midcommisural plane, and $0.45 \mathrm{~mm}$ dorsal to AC/PC plane

Patient 3 had cerebral palsy and underwent DBS of the globus pallidus interna (GPi) for dystonia treatment. Fiber tractography was performed at left lead 0 (Fig. 1a), where facial pulling was noted at $8.0 \mathrm{~V}$. Dystonia improvement was unable to be assessed intraoperatively as the patient was secured to the stereotaxic frame. Therefore, intraoperative therapeutic data were not recorded for this patient. Final coordinates for the tip of the electrode were $15.5 \mathrm{~mm}$ lateral to the $\mathrm{AC} / \mathrm{PC}$ line, $3.31 \mathrm{~mm}$ anterior to the $\mathrm{AC} / \mathrm{PC}$ midcommisural plane, and $2.49 \mathrm{~mm}$ ventral to $\mathrm{AC} / \mathrm{PC}$ plane.

The object creation tool was then used to draw the cortical areas of interest, namely, the premotor, primary motor, supplementary motor, primary sensory, secondary sensory, and frontal eye field cortices based on the Brodmann areas model and the Schaltenbrand-Wahren atlas for definition and confirmation in the iPlan software (BrainLab).
Fiber tractography was performed next using the software's fiber tractography function. Specific electrodes per patient were selected based on the most therapeutic intraoperative and clinical symptom relief. Therefore, patient 1 was evaluated at lead 1, and fiber tracks were centered at $4.25 \mathrm{~mm}$ from the distal end of the lead, and patients 2 and 3 both were evaluated at lead 0 , and fiber tracks were centered at $2.25 \mathrm{~mm}$ from the distal end of the lead (Fig. 1a).

Patients 1 and 3 were analyzed at a fractional anisotropy threshold of 0.2 , while patient 2 was analyzed at 0.45 at the lateral and ventral locations and 0.4 elsewhere. A minimum fiber length of $30 \mathrm{~mm}$ was used for all patients. Volume of interest (VOI) was defined at the region of interest (ROI). VOI cubical $2 \times 2 \times 2 \mathrm{~mm}$ voxels were placed immediately anterior, posterior, lateral, medial, dorsal, and ventral of the desired lead. Each voxel was extended radially to $2 \times 2 \times 4 \mathrm{~mm}, 2 \times 2 \times$ $6 \mathrm{~mm}(2 \times 3 \times 6 \mathrm{~mm}$ for patient 1$)$, and $2 \times 2 \times 8 \mathrm{~mm}(2 \times$ $4 \times 8 \mathrm{~mm}$ for patient 1$)$. Fiber tractography was performed for each dimension and location. The differences in voxel size for patient 1 were due to gantry angle differences during the imaging.

We opted to use this "radial rectangle" method in lieu of simply enlarging the voxel area around the ROI in order to better discern the anatomical location of fibers (Fig. 1b). This method decreased the "noise" that was recorded compared with full-volume ROI tractography. The rectangular enlargement was used to simulate the possible relationship between fiber distance and voltage; larger
Fig. 2 Three-dimensional demonstration of fiber tractography with STN, VIM, and GPi targets in anteroposterior (a), craniocaudal (b), and lateral (c) views. The ROI was a 4-mm cube centered around the targets as calculated from the AC/PC coordinates. There is significant variability in fiber tract targets, particularly between GPi and STN DBS targets
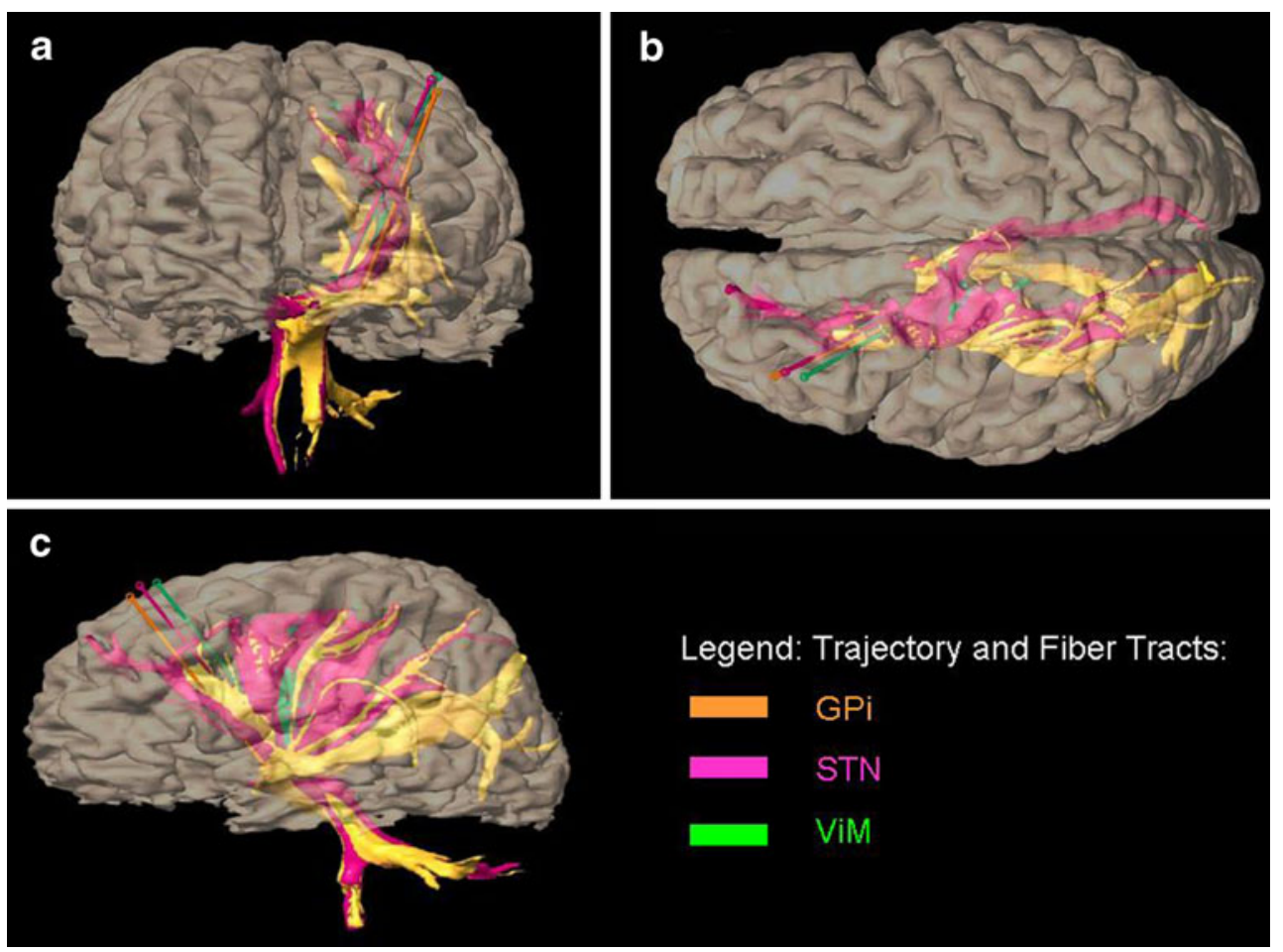
voxels would capture fibers that were stimulated at higher voltages and possibly adverse side effects.

\section{Results}

Fiber tractography demonstrates that, globally, the majority of fiber tracts were medial to GPi, lateral to VIM, and encased in STN. This correlates to known anatomically defined pathways of the internal capsule and the corona radiata. There were varying pathways seen as the region of interest (ROI) was expanded. This is demonstrated with three-dimensional rendition of hemispheric fiber tractography per each DBS target (Fig. 2). Notice the broader spread of fibers passing in proximity of the GPi target (yellow) as compared with STN (pink) and mostly to the specific focallity of the fibers en passage close to the VIM target.

\section{Subthalamic nucleus tractography}

Patient 1, who had undergone DBS placement in the STN, demonstrated fiber tracts leading to the precentral and premotor cortices, particularly at the level of the hand region with a small ROI $(2 \times 2 \times 2 \mathrm{~mm})$. However, additional fiber tracts were incorporated using a large ROI $(2 \times 2 \times 8 \mathrm{~mm})$ laterally, including tracts leading to the prefrontal cortex, the parietal cortex, and the inferior occipitofrontal fasiculus. In addition, the frontal eye fields region (Brodmann area 8) appeared to have approaching tracts (Fig. 3).

\section{Ventral intermediate nucleus tractography}

Patient 2 had undergone DBS placement in the VIM. Tractography demonstrated tracts leading to the motor and premotor cortices with a small ROI. The large ROI tractography demonstrated additional tracts leading to the prefrontal, supplementary motor, and parietal cortices as well as the cerebellum (Fig. 4).

Globus pallidus interna tractography

Patient 3 had a DBS placed in the GPi. Fiber tractography demonstrated tracts leading to the motor, prefrontal, sensory, and parietal cortices when a small ROI was used. The large ROI demonstrated additional tracts leading to the frontal eye fields and the cerebellum (Fig. 5).
Fig. 3 Fiber tractography of lead 1 in patient 1 (STN DBS). Three-dimensional depiction (a) of the small (yellow) and large (purple) ROIs demonstrating increased cortical fiber tract targets. The pink object is the primary motor cortex (based on anatomical landmarks). Axial (b) and coronal $(\mathbf{c}, \mathbf{d})$ slices of source images with color-coded diagrams depict the large ROI fiber tracts (purple-arrows) extending to the prefrontal and parietal cortices, possibly including the frontal eye fields in addition to the fiber tracts of the small ROI (yellow-arrowheads). The pink region is the motor cortex region
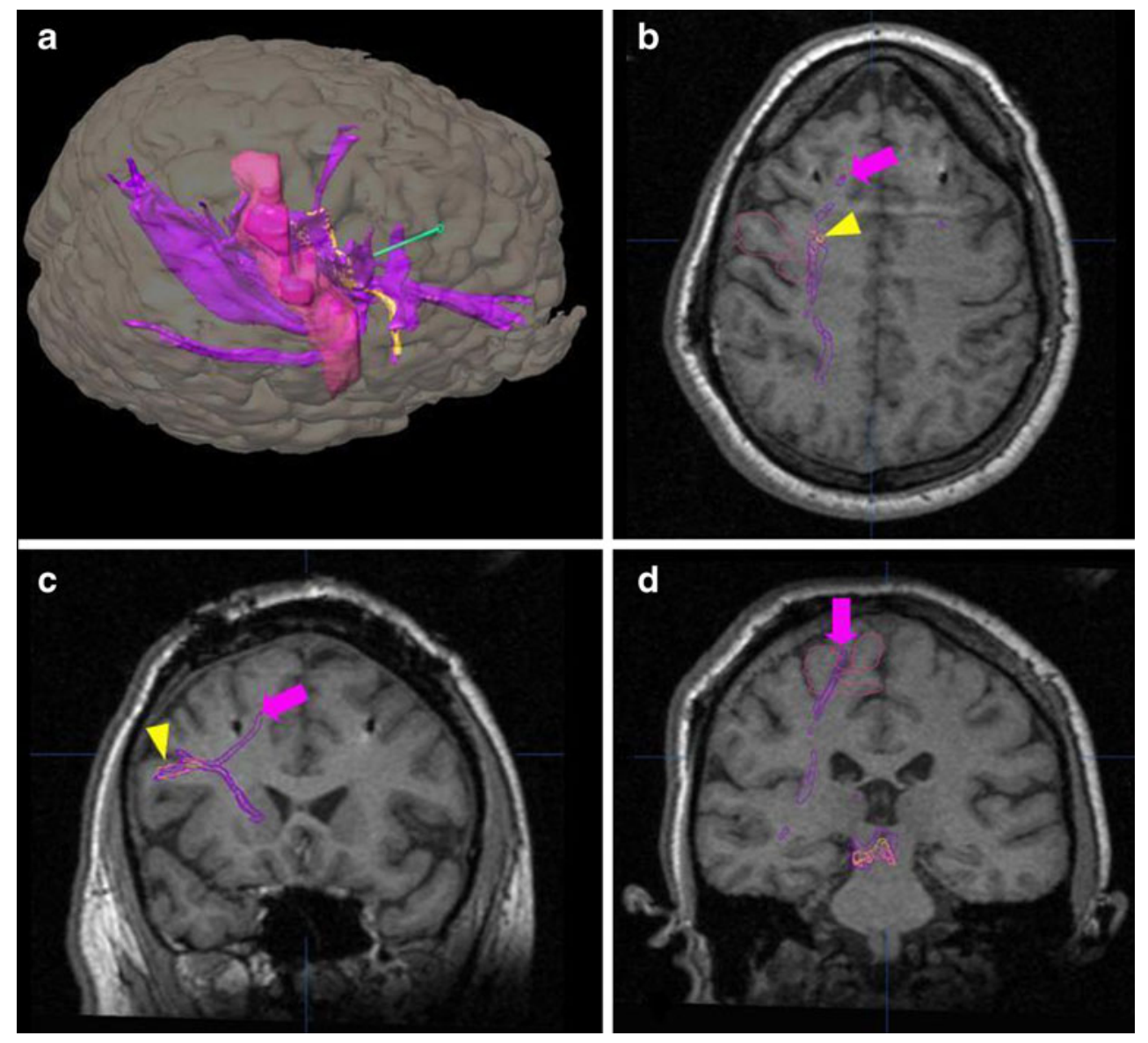
Fig. 4 Fiber tractography of lead 0 in patient 2 (VIM DBS). Three-dimensional depiction (a) of the small (yellow) and large (purple) ROIs demonstrating increased cortical fiber tract targets. The pink object is the primary motor cortex (based on anatomical landmarks). Axial (b) and coronal $(\mathbf{c}, \mathbf{d})$ slices of source images with color-coded diagrams depict the large ROI fiber tracts (purple-arrows) extending to the prefrontal and parietal cortices in addition to the fiber tracts of the small ROI (yellow-arrowhead)
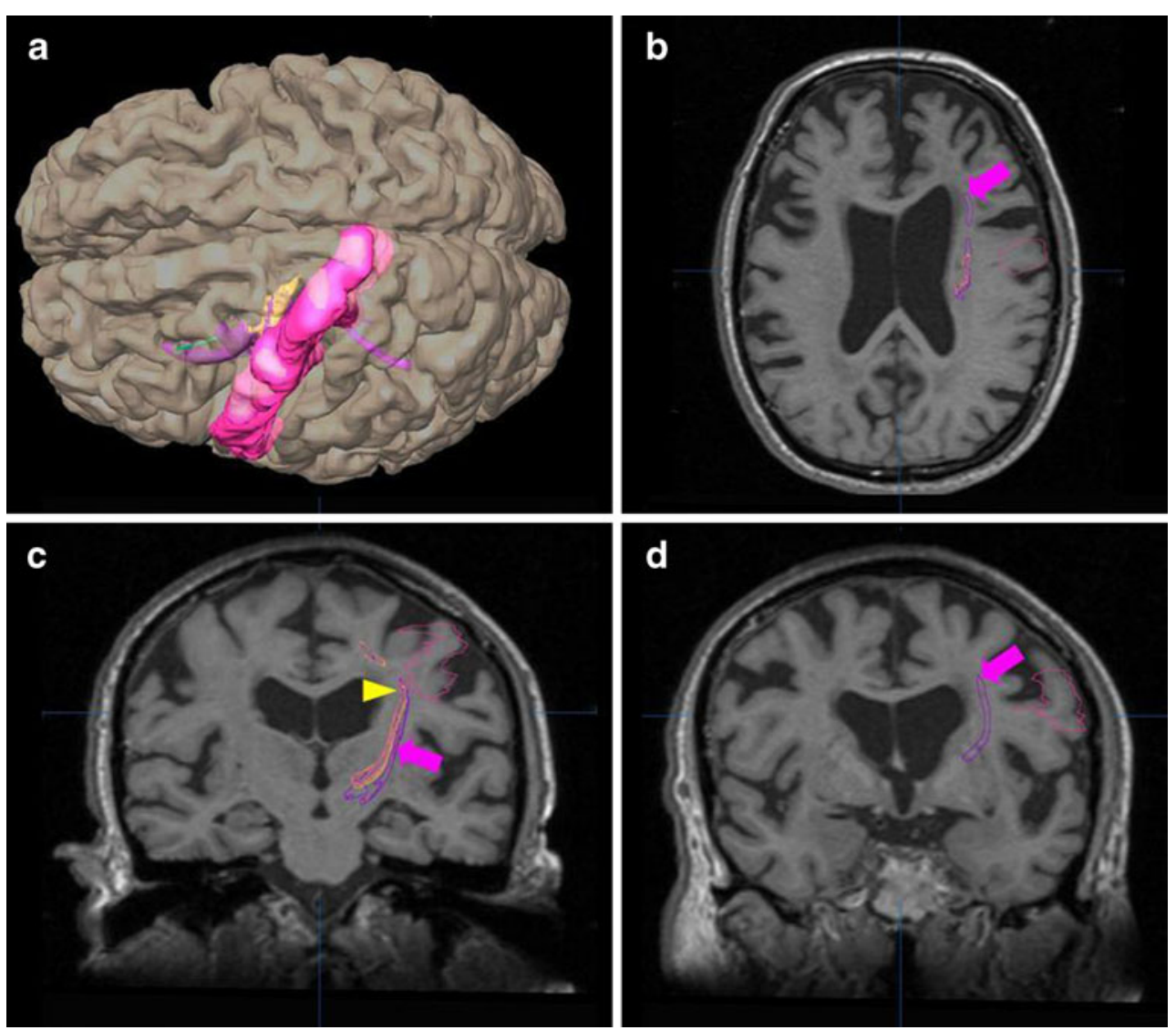

Symptom-tractography correlation

There were no concrete associations with side effects and cortical regions targeted by the "High Voltage" ROI. However, some of the motor side effects (i.e., facial pulling and uncontrolled arm movement) correlated with the fiber tracts leading to the SMA or premotor cortical regions (Table 1).

\section{Discussion}

Diffusion tensor tractography, a method of visualizing white-matter fiber tracts in vivo, has numerous applications in neurosurgery. For DBS, this technology has yielded information beyond what has been previously utilized. Theoretical models have suggested that white-fiber tracts are the limiting factor for side effects during DBS.

For example, Shields et al.[15] demonstrated through clinical data that the common side effect of eye deviation during STN stimulation is due to white-matter fiber tracts being activated in the anterior limb of the internal capsule leading to the frontal eye fields. With the help of voltage vectors and anatomical atlases, the authors diagram a possible model describing the electrical field in relation to the internal capsule. Various other authors have deduced similar anatomical models based on clinical data.[7, 10] With fiber tractography, such anatomical models can be confirmed with MRI. However, variations of electrical field density over anisotropic tissue types (e.g., large diameter axons, small diameter axons, and gray matter) may not allow a direct correlation when compared with tractography.[4, 8]

We have shown in this study the proximity of these white fiber tracts to various leads. Fibers of motor, premotor, supplementary motor, and frontal eye field regions are very intimately associated with STN and GPi stereotactic locations, more so than for VIM. Patient 1 demonstrated intraoperative arm movement as an initial side effect. This may correlate with fiber tracts leading to the hand region of the motor cortex as seen with fiber tractography. Patients 2 and 3 both had facial pulling as intraoperative side effects, which could also be correlated with fiber tracts leading to the face region of the motor cortices. In addition, patient 2 experienced dysarthria intraoperatively, which could be explained by the fibers leading to the prefrontal cortex and to the cerebellum, possibly involving the brainstem (Table 1).

Other known side effects of DBS overstimulation, such as eye deviation and parasthesias, could be explained by the fiber tracts incorporated by the larger ROIs leading to the frontal eye field region of the premotor cortex as well as 
Fig. 5 Fiber tractography of lead 0 in patient 3 (GPi DBS). Three-Dimensional depiction (a) of the small (yellow) and large (purple) ROIs demonstrating increased cortical fiber tract targets. The pink object is the primary motor cortex (based on anatomical landmarks). Coronal (b) and axial (c, d) slices of source images with color-coded diagrams depict the large ROI fiber tracts (purple-arrows) extending to the prefrontal cortex, possibly including the frontal eye fields in addition to the fiber tracts of the small ROI (yellow-arrowheads)
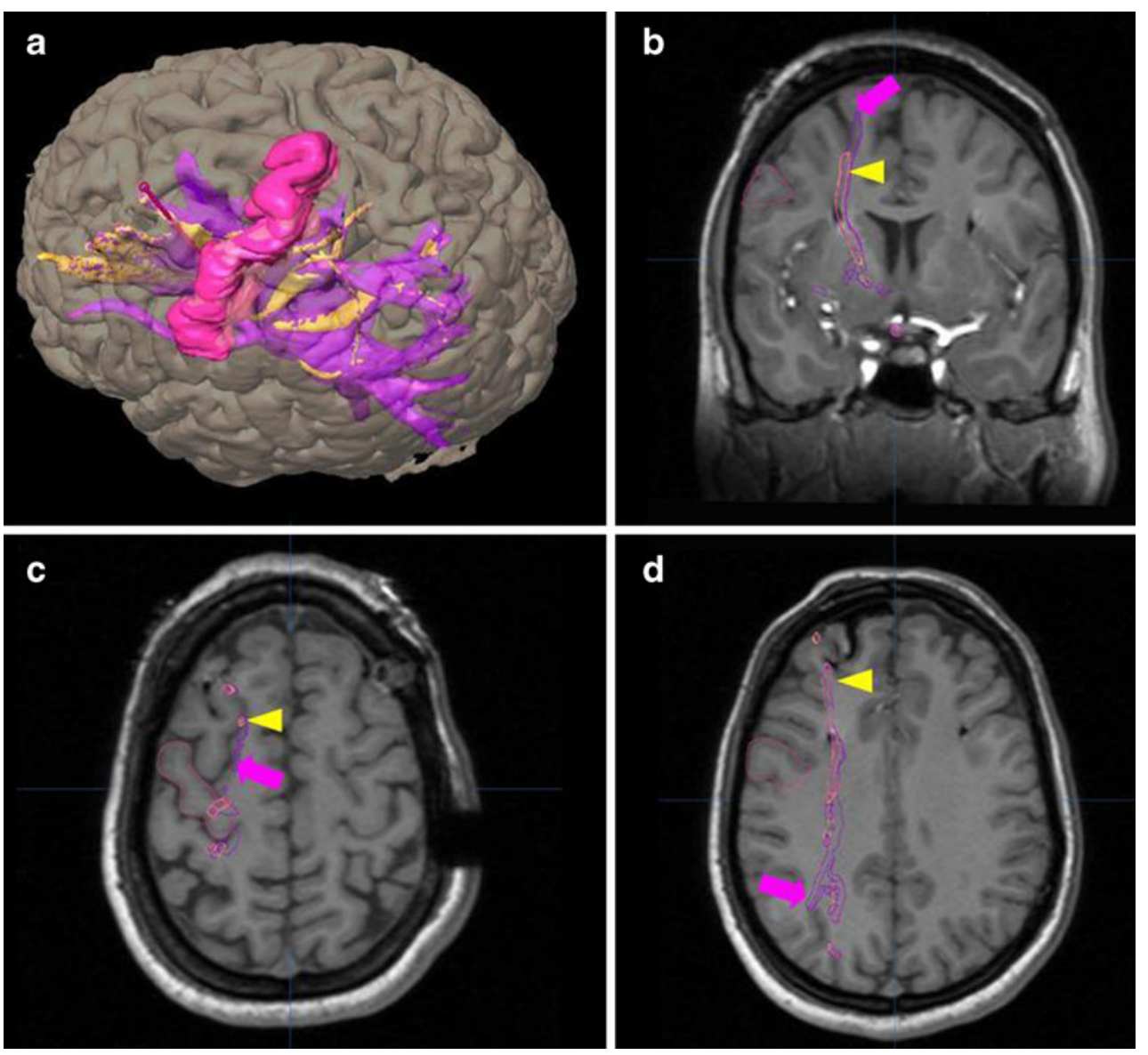

the postcentral gyrus. This finding supports the theory that higher amplitude stimulation leads to recruiting of larger volume of tissue, therefore larger number of fibers, as demonstrated in this fiber tracking study of enlargement of the volume of tracking surrounding the stimulating lead.

Based on the extent of fiber tractography in different targets, one can speculate the level of side effects observed in each target. For example, fiber tractography of the VIM area leads to specific projections to premotor and motor areas, in accordance with the mostly exclusive motor side effects when the VIM is stimulated. Conversely, fiber tractography of the STN and GPi leads to more diffuse spread of fibers throughout the cortex, possibly explaining the multitude of side effects observed when stimulating these targets, including conjugate eye deviation, paresthesias, and mood changes. The latter most likely is related to the projections to prefrontal areas.

Limitations to this preliminary study are related to the DTI image acquisition methods, technical details of the BrainLab tractography software as well as the deduction

Table 1 Motor side effects

\begin{tabular}{|c|c|c|c|c|}
\hline Patient & Target & Initial side effect & Fiber tractography "low voltage" ROI & Fiber tractography "high voltage" ROI \\
\hline 1 & STN & Uncontrolled arm movements & $\begin{array}{l}\text { Primary motor cortex } \\
\text { Premotor cortex }\end{array}$ & $\begin{array}{l}\text { Frontal eye fields } \\
\text { Prefrontal cortex } \\
\text { Parietal cortex }\end{array}$ \\
\hline 2 & VIM & $\begin{array}{l}\text { Facial pulling } \\
\text { Dysarthria }\end{array}$ & $\begin{array}{l}\text { Primary motor cortex } \\
\text { Premotor cortex }\end{array}$ & $\begin{array}{l}\text { Prefrontal cortex } \\
\text { SMA } \\
\text { Parietal cortex }\end{array}$ \\
\hline 3 & GPi & Facial pulling & $\begin{array}{l}\text { Prefrontal cortex } \\
\text { Premotor cortex } \\
\text { Primary motor cortex } \\
\text { Primary sensory cortex }\end{array}$ & $\begin{array}{l}\text { SMA } \\
\text { Parietal cortex }\end{array}$ \\
\hline
\end{tabular}


methods applied to the data. A higher resolution DTI acquisition sequence could allow for smaller ROIs, potentially improving the tractography resolution. In addition, the tractography software was unable to follow tracts that were intersected by large tracts (such as the superior longitudinal fasciculus). This prohibited evaluating tracts leading to the medial superior cortices. In addition, stimulation of thalamic and basal ganglia nuclei could not be characterized with this technique, although they certainly can explain side effect patterns in DBS patients. It is evident that fiber tracts leading to varying cortices explained the side effects in certain patients, but did not explain the absence of other side effects. Perhaps this is due to the varying effects of voltage increases as the field crosses white matter borders. Further studies are necessary to better characterize the above.

Nevertheless, fiber tractography analysis of DBS targets may theoretically impact stereotactic targeting and trajectory planning during DBS placement, perhaps optimizing the treatment of symptoms while reducing the risk for side effects. This study is an initial attempt to develop computerbased imaging models to characterize brain stimulation in its therapeutic and side effect profiles.

Acknowledgements The authors would like to acknowledge the University of California, Los Angeles (UCLA) Ahmanson-Lovelace Brain Mapping Center and the UCLA department of Neurosurgery Operating Department staff. This project was done in accordance of UCLA IRB protocol \#G07-09-091-01.

Open Access This article is distributed under the terms of the Creative Commons Attribution Noncommercial License which permits any noncommercial use, distribution, and reproduction in any medium, provided the original author(s) and source are credited.

\section{References}

1. Basser PJ, Pajevic S, Pierpaoli C, Duda J, Aldroubi A (2000) In vivo fiber tractography using DT-MRI data. Magn Reson Med 44:625-632

2. Bryant JA, De Salles A, Cabatan C, Frysinger R, Behnke E, Bronstein J (2003) The impact of thalamic stimulation on activities of daily living for essential tremor. Surg Neurol 59:478-483
3. De Salles AAF, Frighetto L, Behnke E, Sinha S, Tseng L, Torres R, Lee M, Cabatan-Awang C, Frysinger R (2004) Functional neurosurgery in the MRI environment. Minim Invasive Neurosurg 47:284-289

4. Friston KJ, Harrison L, Penny W (2003) Dynamic causal modelling. Neuroimage 19:1273-1302

5. Gorgulho A, De Salles AAF, Frighetto L, Behnke E (2005) Incidence of hemorrhage associated with electrophysiological studies performed using macroelectrodes and microelectrodes in functional neurosurgery. J Neurosurg 102:888-896

6. Holmes AA, Scollan DF, Winslow RL (2000) Direct histological validation of diffusion tensor MRI in formaldehyde-fixed myocardium. Magn Reson Med 44:157-161

7. Lanotte MM, Rizzone M, Bergamasco B, Faccani G, Melcarne A, Lopiano L (2002) Deep brain stimulation of the subthalamic nucleus: anatomical, neurophysiological, and outcome correlations with the effects of stimulation. Br Med J 72:53

8. Lee L, Friston K, Horwitz B (2006) Large-scale neural models and dynamic causal modelling. Neuroimage 30:1243-1254

9. Lee MWY, De Salles AAF, Frighetto L, Torres R, Behnke E, Bronstein JM (2005) Deep brain stimulation in intraoperative MRI environment - comparison of imaging techniques and electrode fixation methods. Minim Invasive Neurosurg 48:1-6

10. McIntyre CC, Grill WM (2002) Extracellular stimulation of central neurons: influence of stimulus waveform and frequency on neuronal output. J Neurophysiol 88:1592-1604

11. Mobin F, De Salles AAF, Behnke EJ (1999) Correlation between MRI-based stereotactic thalamic deep brain stimulator placement, macroelectrode stimulation and clinical response to tremor control. Stereotact Funct Neurosurg 72:225-232

12. Mori S, Crain BJ, Chacko VP, van Zijl PC (1999) Threedimensional tracking of axonal projections in the brain by magnetic resonance imaging. Ann Neurol 45:265-269

13. Mori S, Kaufmann WE, Davatzikos C, Stieltjes B, Amodei L, Fredericksen K, Pearlson GD, Melhem ER, Solaiyappan M, Raymond GV (2002) Imaging cortical association tracts in the human brain using diffusion-tensor-based axonal tracking. Magn Reson Med 47:215-223

14. Poupon C, Clark CA, Frouin V, Regis J, Bloch I, Le Bihan D, Mangin JF (2000) Regularization of diffusion-based direction maps for the tracking of brain white matter fascicles. Neuroimage 12:184-195

15. Shields DC, Gorgulho A, Behnke E, Malkasian D, Desalles AAF (2007) Contralateral conjugate eye deviation during deep brain stimulation of the subthalamic nucleus. Journal of Neurosurgery 107

16. Stieltjes B, Kaufmann WE, van Zijl PCM, Fredericksen K, Pearlson GD, Solaiyappan M, Mori S (2001) Diffusion tensor imaging and axonal tracking in the human brainstem. Neuroimage 14:723-735 